PROCEEDINGS OF THE

AMERICAN MATHEMATICAL SOCIETY

Volume 127, Number 7, Pages 2045-2052

S 0002-9939(99)04799-1

Article electronically published on February 18, 1999

\title{
TOPOLOGICAL SEQUENCE ENTROPY FOR MAPS OF THE INTERVAL
}

\author{
ROMAN HRIC \\ (Communicated by Mary Rees)
}

\begin{abstract}
A result by Franzová and Smítal shows that a continuous map of the interval into itself is chaotic if and only if its topological sequence entropy relative to a suitable increasing sequence of nonnegative integers is positive. In the present paper we prove that for any increasing sequence of nonnegative integers there exists a chaotic continuous map with zero topological sequence entropy relative to this sequence.
\end{abstract}

\section{INTRODUCTION}

We will be concerned with the space $C(I, I)$ of continuous maps of the interval $I=[0,1]$ into itself. Any map from $C(I, I)$ with positive topological entropy is chaotic, but the converse is not true $[\mathrm{S}]$. Contrary to this topological sequence entropy is a suitable tool for characterizing chaos. In fact, Franzová and Smítal proved the following result.

Theorem 1 ([FS]). A map $f \in C(I, I)$ is chaotic in the sense of Li and Yorke if and only if there is an increasing sequence $T$ of nonnegative integers such that $h_{T}(f)$, the topological sequence entropy of $f$ with respect to $T$, is positive.

There has appeared a natural question whether there is some universal sequence which can be taken in the theorem above. Franzová and Smítal in [FS] conjectured that the sequence $\left(2^{i}\right)_{i=0}^{\infty}$ played the role of this universal sequence. However, the answer to the question is negative. In fact, in the last section we prove

Theorem 6. Let $T$ be an arbitrary increasing sequence of nonnegative integers. Then there is a chaotic map $f \in C(I, I)$ such that $h_{T}(f)=0$.

The paper is organized as follows.

In Preliminaries we present basic notation and terminology and recall the notion of topological sequence entropy. In Theorem 2 we state one known result which will be needed in the sequel. In Section 3 we recall the definition of the adding machine on the Cantor set and consider the map $g$ obtained from the adding machine by the linear extension to the intervals contiguous to the Cantor set. Applying the technique of blowing up orbits to the map $g$ we get a special chaotic map $f$ (depending

Received by the editors May 30, 1997 and, in revised form, October 2, 1997.

1991 Mathematics Subject Classification. Primary 26A18, 54H20, 58F13.

Key words and phrases. Adding machine, blowing up orbits, chaotic map, topological sequence entropy.

The author has been partially supported by the Slovak grant agency, grant number 1/1470/94.

(C)1999 American Mathematical Society 
on the point $q$ whose orbit is blown up), and we study the properties of this map with respect to topological sequence entropy (see Theorem 3 and Corollary 4). In Section 4, for any increasing sequence $T$ of nonnegative integers we choose the point $q$ in a special way, and in Lemma 5 we find an upper bound for the span of the map $f$ relative to the sequence $T$. Using this upper bound we finally prove Theorem 6 .

\section{Preliminaries}

Let $\mathbb{Z}$ denote the set of all integers, $\mathbb{N}$ the set of all positive integers. For $f \in C(I, I), f^{n}$ denotes the $n$-th iterate of $f$ and $\omega_{f}(x)$ denotes the $\omega$-limit set of the trajectory $\left(f^{n} x\right)_{n=0}^{\infty}$ of $x . \operatorname{FOrb}_{f}(x)=\left\{y \in I ; \exists i, j \in \mathbb{N}\right.$ with $\left.f^{i} y=f^{j} x\right\}$ is called the full orbit of $x$ and for an interval $J \subseteq I, \operatorname{Orb}_{f}(J)=\left\{f^{n} J ; n=0,1, \ldots\right\}$ is called the orbit of $J$. A periodic point of $f$ is any $x \in I$ such that $f^{n} x=x$ for some $n \in \mathbb{N}$, the smallest such $n$ is called the period of $x$; analogously for an interval $J \subseteq I$ satisfying $f^{n} J=J$ for some $n$. The set of all periodic points of $f$ is denoted by $\operatorname{Per}(f)$. A map $f$ is chaotic (in the sense of Li and Yorke, see [LY]), if there is an uncountable set $S$ such that for any $x, y \in S, x \neq y$, and for any $p \in \operatorname{Per}(f)$

$$
\begin{gathered}
\limsup _{n \rightarrow \infty}\left|f^{n} x-f^{n} y\right|>0, \\
\liminf _{n \rightarrow \infty}\left|f^{n} x-f^{n} y\right|=0, \\
\limsup _{n \rightarrow \infty}\left|f^{n} x-f^{n} p\right|>0 .
\end{gathered}
$$

An extensive list of equivalent conditions can be found in [FŠS]. A map is called nonchaotic if it is not chaotic. If $T=(t(i))_{i=1}^{\infty}$ is an arbitrary increasing sequence of nonnegative integers define the $T$-trajectory of $x$ to be the sequence $\left(f^{t(i)} x\right)_{i=1}^{\infty}$.

Definition ([G]). Let $f \in C(I, I)$ and $T$ be an arbitrary sequence of nonnegative integers.

A set $E \subseteq I$ is said to be $(T, f, \varepsilon, n)$-separated if for any $x, y \in E, x \neq y$ there is an index $i, 1 \leq i \leq n$ such that $\left|f^{t(i)} x-f^{t(i)} y\right|>\varepsilon$. Let $\operatorname{Sep}(T, f, \varepsilon, n)$ denote the largest of cardinalities of all $(T, f, \varepsilon, n)$-separated sets. Put $\operatorname{Sep}(T, f)=$ $\lim _{\varepsilon \rightarrow 0} \limsup _{n \rightarrow \infty} \frac{1}{n} \log \operatorname{Sep}(T, f, \varepsilon, n)$.

A set $F \subseteq I$ is said to be a $(T, f, \varepsilon, n)$-span if for any $x \in I$ there is some $y \in F$ such that $\left|f^{t(i)} x-f^{t(i)} y\right|<\varepsilon$ for $1 \leq i \leq n$. Let $\operatorname{Span}(T, f, \varepsilon, n)$ denote the smallest of cardinalities of all $(T, f, \varepsilon, n)$-spans. Put

$$
\operatorname{Span}(T, f)=\lim _{\varepsilon \rightarrow 0} \limsup _{n \rightarrow \infty} \frac{1}{n} \log \operatorname{Span}(T, f, \varepsilon, n) .
$$

Then $\operatorname{Sep}(T, f)=\operatorname{Span}(T, f)$ (see $[\mathrm{G}]$ ) and we define the topological sequence entropy of $f$ relative to $T, h_{T}(f)$, to be $\operatorname{Sep}(T, f)$. If $t(i)=i-1, i=1,2, \ldots$ then $h_{T}(f)$ is the topological entropy $h(f)$ of $f$.

Remark. Analogously we can define topological sequence entropy for continuous maps of any compact metric space $X$ into itself. Topological sequence entropy can be viewed as the topological entropy of the nonautonomous dynamical system given on the space $X$ by the sequence of maps $f^{t(1)}, f^{t(2)-t(1)}, f^{t(3)-t(2)}, \ldots$ (see $[\mathrm{KS}]$ ).

Theorem 2 (see [S, Theorem 3.5]). Let $f \in C(I, I), h(f)=0$ and $\omega$ be an infinite $\omega$-limit set of $f$. Then there is a sequence $(J(i))_{i=1}^{\infty}$ of periodic intervals of $f$ with the following properties: 
(i) $J(i)$ has period $2^{i}$,

(ii) $J(i+1) \cup f^{2^{i}} J(i+1) \subseteq J(i)$,

(iii) $\omega \subseteq \cup \operatorname{Orb}_{f}(J(i))$.

When using this theorem we will always assume the intervals $J(i)$ to be minimal.

\section{BLOWING UP THE ADDING MACHINE}

In the sequel we will use the concept of the adding machine (see $[\mathrm{GH}],[\mathrm{M}]$, cf. also $[\mathrm{BS}])$.

Define $X=\{0,1\}^{\mathbb{N}}$ to be the set of all sequences of two symbols and $X \mid m=$ $\{0,1\}^{\{1, \ldots, m\}}$ for $m \in \mathbb{N}$. For $\alpha \in X$ and $m \in \mathbb{N}$ we put $\alpha \mid m=(\alpha(1), \ldots, \alpha(m)) \in$ $X \mid m$. Put $\operatorname{CS}(0)=\left[0, \frac{1}{3}\right], \operatorname{CS}(1)=\left[\frac{2}{3}, 1\right]$ and $\operatorname{CS}(00)=\left[0, \frac{1}{9}\right], \operatorname{CS}(01)=\left[\frac{2}{9}, \frac{1}{3}\right]$, $\mathrm{CS}(10)=\left[\frac{2}{3}, \frac{7}{9}\right], \operatorname{CS}(11)=\left[\frac{8}{9}, 1\right]$, etc. Then we have for the Cantor set CS

$$
\mathrm{CS}=\bigcap_{m=1}^{\infty} \bigcup_{\alpha \in X \mid m} \operatorname{CS}(\alpha) .
$$

This determines in a natural way the bijection $\varkappa$ between CS and $X$ : assign to $q \in \mathrm{CS}$ the element $\varkappa(q) \in X$ for which $q \in \mathrm{CS}(\varkappa(q) \mid m)$ for every $m \in \mathbb{N}$. We call $\varkappa(q)$ the code of $q$ and we also write $\varkappa(q)=(q(i))_{i=1}^{\infty}$. Now we can define the adding machine Add: CS $\rightarrow$ CS by

$$
\operatorname{Add}(q)=\varkappa^{-1}(\varkappa(q)+1)
$$

where the addition is modulo 2 from the left to the right. The map Add is a bijection.

Completing the adding machine linearly on each interval contiguous to CS we get the continuous function $g: I \rightarrow I$ which then satisfies the following properties (see [BS, Section 4]):

(g1) CS is the unique infinite $\omega$-limit set of $g$,

(g2) $g$ is nonchaotic (and so $h(g)=0$ ),

(g3) $\forall x \in I$ : $\operatorname{Card} g^{-1}(x) \leq 3$.

Now fix an arbitrary $q \in \mathrm{CS}$ such that both symbols 0 and 1 occur in its code $\varkappa(q)$ infinitely many times. Due to (g3) we can use the technique of blowing up orbits (see $[\mathrm{H}]$, cf. also $[\mathrm{BS}]$ ).

$\operatorname{FOrb}_{g}(q)$ is countable and in the following we take its enumeration in the form $\operatorname{FOrb}_{g}(q)=\left\{q_{k} ; k \in K\right\}$ where $q_{i}=\operatorname{Add}^{i}(q)$ for every $i \in \mathbb{Z}$ and $K \supseteq \mathbb{Z}$ is a suitable countable index set. Let $\left\{Q_{k} ; k \in K\right\}$ be a system of compact pairwise disjoint subintervals of $I$ with similar ordering as the set $\left\{q_{k} ; k \in K\right\}$ (i.e., $Q_{k}<Q_{l}$ iff $q_{k}<q_{l}$ ). Now replace every $q_{k}$ by $Q_{k}$ and compress the rest of the interval $I$ in such a way that we again receive $I$. Formally, apply to $I$ the blowing up morphism $\varphi$, which is an increasing set-valued function assigning to any $q_{k}$ the interval $Q_{k}$ and to any $x \in I \backslash \operatorname{FOrb}_{g}(q)$ a point such that $\varphi(I)=I$ and $\varphi$ is linear on every interval contiguous to $\operatorname{FOrb}_{g}(q)$. The map $\varphi$ has an inverse $\varphi^{-1}$ which is a continuous real-valued nondecreasing function in $C(I, I)$, constant on every $Q_{k}$. 
Define $f \in C(I, I)$ by

$$
f(x)= \begin{cases}\varphi\left(g\left(\varphi^{-1}(x)\right)\right) & \text { for } x \in I \backslash \bigcup_{k \in K} Q_{k}, \\ \text { linearly extended } & \text { on } \bigcup_{k \in K} Q_{k} .\end{cases}
$$

Then $f$ has the following properties (see [BS, Theorem 6.2]):

(f1) $f$ has the unique infinite $\omega$-limit set $\omega=\varphi(\mathrm{CS}) \backslash \bigcup_{k \in K} \operatorname{Int} Q_{k}$,

(f2) $\omega \varsubsetneqq \bigcap_{i=1}^{\infty} \cup \operatorname{Orb} J(i)$

(f3) $h(f)=0$.

Associate with $q$ the increasing sequence $A(q)=(a(i))_{i=1}^{\infty}$ of all indices in which the code of $q$ "changes" the symbols, i.e., $q(a(i)+1)=1-q(a(i))$. Using this sequence, define another one $B(q)=(b(i))_{i=1}^{\infty}$ by putting $b(i)=2^{a(1)}+\cdots+2^{a(i)}$ for every $i \in \mathbb{N}$.

Theorem 3. For $f$ and $B$ defined above we have $h_{B}(f)>0$.

Proof. Let $(J(i))_{i=1}^{\infty}$ be the sequence of periodic intervals of $f$ from Theorem 2 applied to $f$ and $\omega$, such that $Q_{0} \subseteq J(i)$ for every $i \in \mathbb{N}$. In the interval $J(i)$ there is the unique fixed point of $f^{2^{i}}$, denote it by fix $(i)$. Let fix $(0)$ be the unique fixed point of $f$. Define $L(i)(R(i)$, respectively) to be the left (right, respectively) component of $\operatorname{Conv}(J(a(i))$, fix $(a(i)-1)) \backslash \operatorname{Int} Q_{0}$ for every $i \in \mathbb{N}$ (here Conv $A$ denotes the convex hull of $A$ ).

First we prove that

$$
\begin{aligned}
& f^{2^{a(i)}} L(i) \supseteq L(i+1) \cup R(i+1), \\
& f^{2^{a(i)}} R(i) \supseteq L(i+1) \cup R(i+1) .
\end{aligned}
$$

To this end we first realize that

$$
\begin{aligned}
\operatorname{Conv}(J(a(i)+1), \operatorname{fix}(a(i))) & \supseteq \operatorname{Conv}(J(a(i+1)), \operatorname{fix}(a(i+1)-1)) \\
& \supseteq L(i+1) \cup R(i+1) .
\end{aligned}
$$

Without loss of generality we can assume that $q(a(i))=0$. Then $\operatorname{fix}(a(i)) \in L(i)$ and $f^{2^{a(i)}} J(a(i)+1) \subseteq L(i)$. To prove (1) it suffices to use $f^{2^{a(i)}} \operatorname{fix}(a(i))=\operatorname{fix}(a(i))$, $f^{2^{a(i)}}\left(f^{2^{a(i)}} J(a(i)+1)\right)=J(a(i)+1)$ and $(3)$.

From $J(a(i)+1) \cap R(i) \neq \emptyset$ we have $f^{2^{a(i)}} J(a(i)+1) \cap f^{2^{a(i)}} R(i) \neq \emptyset$. To prove (2) it is sufficient to realize that $f^{2^{a(i)}} \operatorname{fix}(a(i)-1)=\operatorname{fix}(a(i)-1) \in R(i)$, $f^{2^{a(i)}} J(a(i)+1)$ lies to the left from fix $(a(i))$ and to use (3).

Now fix $n \in \mathbb{N}$ and $0<\varepsilon<\operatorname{diam} Q_{0}$. We are going to show that $\operatorname{Sep}(B, f, \varepsilon, n) \geq$ $2^{n}$. To see this, take any sequence $(S(1), \ldots, S(n))$ where $S(i)$ is either $L(i+1)$ or $R(i+1), i=1, \ldots, n$. By (1) and (2) there is a point $x \in I$ such that $f^{b(i)} x \in S(i)$ for $i=1, \ldots, n$. There are $2^{n}$ such sequences and so we have $2^{n}$ different points forming an $(B, f, \varepsilon, n)$-separated set (note that $\operatorname{dist}(L(i), R(i))>\varepsilon$ for any $i)$. Hence $\operatorname{Sep}(B, f, \varepsilon, n) \geq 2^{n}$. Since $0<\varepsilon<\operatorname{diam} Q_{0}$ and $n \in \mathbb{N}$ were arbitrary, we get $h_{B}(f) \geq \log 2$.

Corollary 4. The map $f$ defined above is chaotic.

Remark. If we take $q$ with $\varkappa(q)$ almost stationary we obtain a nonchaotic map which has the unique infinite $\omega$-limit set, and satisfies (f2) and (f3) but not (f1). 


\section{The MAIN RESULT}

Let $T=(t(i))_{i=1}^{\infty}$ be an arbitrary increasing sequence of nonnegative integers. Take any point $q \in \mathrm{CS}$ such that for the sequence $A(q)=(a(i))_{i=1}^{\infty}$ (defined in the previous section) it holds that

$$
2^{a(i)}>t\left(2^{i}\right) \text { for } i=1,2, \ldots
$$

Let $f$ be the function in $C(I, I)$ obtained from $g$ by blowing up the full orbit $\mathrm{FOrb}_{g}(q)$ as in the previous section.

If $m \in \mathbb{N}$ and $\mathcal{S}=\left\{S_{1}, \ldots, S_{s}\right\}$ is a nonempty finite family of pairwise disjoint nonempty subsets of $I$ then $\operatorname{Code}(m, \mathcal{S})$ will denote the set of all sequences $\left(A_{1}, \ldots, A_{m}\right) \in \mathcal{S}^{m}$ with the property that there exists a point $x \in I$ such that $f^{t(i)} x \in A_{i}, i=1, \ldots, m$. In such a case we will say that $\left(A_{1}, \ldots, A_{m}\right)$ is the code of $x$ (or, more precisely, the code of $x$ of length $m$ with symbols from $\mathcal{S}$ ).

Lemma 5. For any $\varepsilon>0$ there exists a periodic interval $J(a(p))$ of $f$ such that $\operatorname{Span}\left(T, f \mid \cup \operatorname{Orb}_{f} J(a(p)), \varepsilon, 2^{n}\right)=O\left(2^{n^{2}}\right)$.

Proof. In the sequel $J_{j}(i)$, where $-2^{i-1}+1 \leq j \leq 2^{i-1}$, will denote that interval in $\operatorname{Orb}_{f} J(i)$ which contains $Q_{j}$. Let $\varepsilon$ be an arbitrary positive number. There is a $p \in \mathbb{N}$ such that the periodic interval $J(a(p))$ satisfies

$$
\operatorname{diam} J_{j}(a(p))-\operatorname{diam} Q_{j}<\varepsilon \quad \text { for } j=-2^{a(p)-1}+1, \ldots,-1,0,1, \ldots, 2^{a(p)-1} .
$$

To see this, suppose this is not the case. Then there is a nested sequence $(A(n))_{n=0}^{\infty}$ of periodic intervals $A(n) \in \operatorname{Orb} J(n)$ such that $\bigcap_{n=1}^{\infty} A(n)$ is an interval with diameter larger than the diameter of that of the intervals $Q_{k}, k \in K$, which is contained in it. But then the map $g$ has a nested sequence $\left(\varphi^{-1} A(n)\right)_{n=1}^{\infty}$ of periodic intervals whose intersection is not a point which is impossible.

Let $n$ be an arbitrary positive integer. Put

$$
\begin{aligned}
& M_{j}(i)=J_{j}(a(p+i-1)) \backslash J_{j}(a(p+i)) \text { for } i=1, \ldots, n, \\
& \quad j=-2^{a(p)-1}+1, \ldots,-1,0,1, \ldots, 2^{a(p)-1} .
\end{aligned}
$$

It is easy to see that the defined intervals have the following property:

any periodic interval from $\operatorname{Orb}_{f} J(a(p+r)), 1 \leq r \leq n$

either is one of the intervals $J_{-2^{a(p)-1}+1}(a(p+r)), \ldots, J_{2^{a(p)-1}}(a(p+r))$,

or is contained in some $M_{j}(s), 1 \leq s \leq r$.

Put $\mathcal{J}=\left\{J_{-2^{a(p)-1}+1}(a(p+n)), \ldots, J_{2^{a(p)-1}}(a(p+n))\right\}, \mathcal{M}=\bigcup_{j}\left\{M_{j}(1), \ldots\right.$, $\left.M_{j}(n)\right\}$ and consider the decomposition $\mathcal{D}=\mathcal{M} \cup \mathcal{J}$ of the set $\cup \mathrm{Orb}_{f} J(a(p))$. To each $x \in \cup \operatorname{Orb}_{f} J(a(p))$ assign its code $\left(c(1), \ldots, c\left(2^{n}\right)\right) \in \operatorname{Code}\left(2^{n}, \mathcal{D}\right)$. From (4) it follows that any such code can contain at most $2^{a(p)}$ symbols from $\mathcal{J}$. Suppose that a code $c$ contains a symbol from $\mathcal{J}$ and let $k$ is the first number such that $c(k)$ belongs to $\mathcal{J}$. Then the rest of the code $c$, i.e. $\left(c(k+1), \ldots, c\left(2^{n}\right)\right)$ is uniquely determined. This straightforwardly follows from (5). Thus

$$
\operatorname{Card} \operatorname{Code}\left(2^{n}, \mathcal{D}\right) \leq \sum_{m=0}^{2^{n}} \operatorname{Card} \operatorname{Code}(m, \mathcal{M}) \cdot \operatorname{Card} \mathcal{J}
$$

We claim that if $c=\left(M_{k_{1}}\left(c_{1}\right), \ldots, M_{k_{m}}\left(c_{m}\right)\right) \in \operatorname{Code}(m, \mathcal{M})$ and $c_{i}>c_{j}$ for some $1 \leq i<j \leq m$ then $M_{k_{j}}\left(c_{j}\right)$ is uniquely determined by $\left(M_{k_{1}}\left(c_{1}\right), \ldots, M_{k_{i}}\left(c_{i}\right)\right)$. 
In fact, the existence of a point with code $c$ shows that $f^{t(i)-t(j)} M_{k_{i}}\left(c_{i}\right) \cap M_{k_{j}}\left(c_{j}\right) \neq$ $\emptyset$. Further, $M_{k_{i}}\left(c_{i}\right) \subseteq J_{k_{i}}\left(a\left(p+c_{i}-1\right)\right)$. Using $c_{i}-1 \geq c_{j}$, (5) then implies $f^{t(j)-t(i)} M_{k_{i}}\left(c_{i}\right) \subseteq M_{k_{j}}\left(c_{j}\right)$.

Note that the indices $k_{2}, \ldots, k_{m}$ are uniquely determined by $k_{1}$. Now fix an index $k_{1}$ and to each code $c=\left(M_{k_{1}}\left(c_{1}\right), \ldots, M_{k_{m}}\left(c_{m}\right)\right) \in \operatorname{Code}(m, \mathcal{M})$ assign a nondecreasing sequence ris $(c)$, the rising sun sequence of $c$, defined by $\operatorname{ris}(c)(i)=$ $\max \{c(1), \ldots, c(i)\}, i=1, \ldots, m$. By what we showed above, if $\left(M_{k_{1}}\left(c_{1}\right), \ldots\right.$, $\left.M_{k_{i-1}}\left(c_{i-1}\right), M_{k_{i}}\left(c_{i}\right)\right)$ and $\left(M_{k_{1}}\left(c_{1}\right), \ldots, M_{k_{i-1}}\left(c_{i-1}\right), M_{k_{i}}\left(d_{i}\right)\right)$ are two codes with $c_{i} \neq d_{i}$ then $c_{i}, d_{i} \geq c_{1}, \ldots, c_{i-1}$. This implies that $\operatorname{ris}(c) \neq \operatorname{ris}(d)$ whenever $c, d$ are two different codes from $\operatorname{Code}(m, \mathcal{M})$ with the same index $k_{1}$. When we realize that there are $2^{a(p)}$ possible values for $k_{1}$ we can bound above $\operatorname{Card} \operatorname{Code}(m, \mathcal{M})$ by the number of all nondecreasing sequences $\{1, \ldots, m\} \rightarrow\{1, \ldots, n\}$, which equals $\left(\begin{array}{c}m+n-1 \\ m\end{array}\right) \leq\left(\begin{array}{c}2^{n}+n-1 \\ 2^{n}\end{array}\right)$, multiplied by $2^{a(p)}$. Hence, by $(6)$

$$
\begin{aligned}
\operatorname{Card} \operatorname{Code}\left(2^{n}, \mathcal{D}\right) & \leq \sum_{m=0}^{2^{n}}\left(\begin{array}{c}
m+n-1 \\
m
\end{array}\right) \cdot 2^{a(p)} \cdot 2^{a(p)} \\
& \leq\left(2^{n}+1\right) \cdot\left(\begin{array}{c}
2^{n}+n-1 \\
2^{n}
\end{array}\right) \cdot 2^{2 \cdot a(p)}=O\left(2^{n^{2}}\right) .
\end{aligned}
$$

Finally, fix an $N \in \mathbb{N}$ such that $1 / N<\varepsilon$ and cut each $J_{j}(a(p+n))$ to $N$ equally long intervals $K_{j}(1), \ldots, K_{j}(N)$ and put $\mathcal{E}=\bigcup_{j}\left\{K_{j}(1), \ldots, K_{j}(N)\right\} \cup \mathcal{M}$. Since any code from $\operatorname{Code}\left(2^{n}, \mathcal{D}\right)$ contains at most $2^{a(p)}$ symbols from $\mathcal{J}$ we have

$$
\operatorname{Card} \operatorname{Code}\left(2^{n}, \mathcal{E}\right) \leq \operatorname{Card} \operatorname{Code}\left(2^{n}, \mathcal{D}\right) \cdot N^{2^{a(p)}}=O\left(2^{n^{2}}\right)
$$

It is obvious that $\operatorname{Span}\left(T, f \mid \cup \operatorname{Orb}_{f} J(a(p)), \varepsilon, 2^{n}\right) \leq \operatorname{Card} \operatorname{Code}\left(2^{n}, \mathcal{E}\right)$. In fact, if for each code from $\operatorname{Code}\left(2^{n}, \mathcal{E}\right)$ we choose one point having this code then the set of all chosen points is an $\left(T, f \mid \cup \operatorname{Orb}_{f} J(a(p)), \varepsilon, 2^{n}\right)$-span.

Theorem 6. Let $T$ be an arbitrary increasing sequence of nonnegative integers. Then there is a chaotic map $f \in C(I, I)$ such that $h_{T}(f)=0$.

Proof. For $T$ define $f$ as in the beginning of this section. Fix $\varepsilon>0$. We will show that $\limsup _{n \rightarrow \infty} \frac{1}{n} \log \operatorname{Span}(T, f, \varepsilon, n)=0$.

Let $J(a(p))$ be the interval from Lemma 5 . To bound above $\operatorname{Span}\left(T, f, \varepsilon, 2^{n}\right)$ we use Lemma 5 but we have to take into account also $T$-trajectories starting in intervals contiguous to $\operatorname{Orb} J(a(p))$, i.e. in intervals from the family $\mathcal{C}=\left\{C(1), C_{1}(2)\right.$, $\left.C_{2}(2), \ldots, C_{1}(a(p)), \ldots, C_{2^{a(p)-1}}(a(p))\right\}$. Here $C(1)$ is contiguous to $\operatorname{Orb}_{f} J(1)$, then intervals $C_{1}(2), C_{2}(2)$ are remaining two contiguous intervals to $\operatorname{Orb}_{f} J(2)$, etc. Evidently, $i>j$ implies $f^{k} C_{r}(i) \cap C_{s}(j)=\emptyset$ for any $k \in \mathbb{N}, 1 \leq r \leq 2^{j-1}$ and $1 \leq s \leq 2^{i-1}$. A code from $\operatorname{Code}(m, \mathcal{C})$ is a finite sequence of the form

$$
\begin{gathered}
\left(C_{r\left(i_{1}, 1\right)}\left(i_{1}\right), \ldots, C_{r\left(i_{1}, m_{1}\right)}\left(i_{1}\right), C_{r\left(i_{2}, 1\right)}\left(i_{2}\right), \ldots, C_{r\left(i_{2}, m_{2}\right)}\left(i_{2}\right), \ldots\right. \\
\left.\ldots, C_{r\left(i_{k}, 1\right)}\left(i_{k}\right), \ldots, C_{r\left(i_{k}, m_{k}\right)}\left(i_{k}\right)\right)
\end{gathered}
$$

where $m_{1}+\cdots+m_{k}=m$ and $i_{1}<\ldots<i_{k}$. We are going to estimate $\operatorname{Card} \operatorname{Code}(m, \mathcal{C})$.

Obviously, $k \leq m$ and $i_{1}<\cdots<i_{k} \leq a(p)$. Hence $k \leq \min \{m, a(p)\}$. Fix $k, m_{1}, \ldots, m_{k}, i_{1}, \ldots, i_{k}$. First, realize that each subsequence $\left(C_{r\left(i_{j}, 1\right)}\left(i_{j}\right), \ldots\right.$, $\left.C_{r\left(i_{j}, m_{j}\right)}\left(i_{j}\right)\right)$ is uniquely determined by $C_{r\left(i_{j}, 1\right)}\left(i_{j}\right)$. This follows from the fact that for any $l \in \mathbb{N}, 1 \leq i \leq a(p), 1 \leq r \leq 2^{i-1}$ there is exactly one $s, 1 \leq s \leq 2^{i-1}$, 
such that $f^{l} C_{r}(i) \cap C_{s}(i) \neq \emptyset$. Since there are $2^{i_{j}-1}$ possibilities for the choice of $C_{r\left(i_{j}, 1\right)}\left(i_{j}\right)$, for fixed $k, m_{1}, \ldots, m_{k}, i_{1}, \ldots, i_{k}$ we obtain at most $2^{i_{1}-1} \cdots \cdots 2^{i_{k}-1}=$ $2^{i_{1}+\cdots+i_{k}-k} \leq 2^{a(p)^{2}}$ sequences of the form (8).

There are $\left(\begin{array}{c}m-1 \\ k-1\end{array}\right)$ ways how to write $m$ in the form $m_{1}+\cdots+m_{k}$ and $\left(\begin{array}{c}a(p) \\ k\end{array}\right)$ ways of chosing $k$ positive integers $i_{1}<\cdots<i_{k} \leq a(p)$. From all this we obtain that

$$
\begin{aligned}
\operatorname{Card} \operatorname{Code}(m, \mathcal{C}) & \leq \sum_{k=1}^{\min \{m, a(p)\}}\left(\begin{array}{c}
m-1 \\
k-1
\end{array}\right) \cdot\left(\begin{array}{c}
a(p) \\
k
\end{array}\right) \cdot 2^{a(p)^{2}} \\
& \leq 2^{n} \cdot 2^{n \cdot(a(p)-1)} \cdot a(p) ! \cdot 2^{a(p)^{2}}=O\left(2^{a(p) \cdot n}\right) .
\end{aligned}
$$

Cut each interval from the family $\mathcal{C}$ into $N$ equally long intervals and denote by $\mathcal{C}_{c u t}$ the family of all intervals obtained in such a way. We are going to estimate Card $\operatorname{Code}\left(m, \mathcal{C}_{c u t}\right)$. Take any $j \in\{1, \ldots, k\}$ and consider all points having the code $\left(C_{r\left(i_{j}, 1\right)}\left(i_{j}\right), \ldots, C_{r\left(i_{j}, m_{j}\right)}\left(i_{j}\right)\right)$, a subcode of $(8)$. The number of different codes from $\operatorname{Code}\left(m_{j}, \mathcal{C}_{c u t}\right)$ belonging to the considered points is at most $N+\left(m_{j}-1\right) \cdot(N-1) \leq$ $m_{j} \cdot N$. This follows from the fact that $f$ is strictly monotone on each $C_{r}(i)$ which implies that $f^{-l} C_{r}(i) \cap C_{s}(i)$ is either an interval or the empty set and in the former case $f^{l}$ is strictly monotone on this interval. Therefore the number of different codes from $\operatorname{Code}\left(m, \mathcal{C}_{c u t}\right)$ belonging to all points whose code from $\operatorname{Code}(m, \mathcal{C})$ is the sequence (8) is at most $m_{1} \cdot N \cdots \cdots m_{k} \cdot N \leq 2^{a(p) \cdot n} \cdot N^{a(p)}$. Since this upper bound does not depend on the integers $k, m_{1}, \ldots, m_{k}, i_{1}, \ldots, i_{k}$, from (8) we have, by (9),

$$
\operatorname{Card} \operatorname{Code}\left(m, \mathcal{C}_{c u t}\right) \leq 2^{a(p) \cdot n} \cdot N^{a(p)} \cdot \operatorname{Card} \operatorname{Code}(m, \mathcal{C})=O\left(2^{2 \cdot a(p) \cdot n}\right) .
$$

Now we can bound above the number of all codes from $\operatorname{Code}\left(2^{n}, \mathcal{E} \cup \mathcal{C}_{c u t}\right)$. Each such a code consists of a (possibly empty) block of symbols from $\mathcal{C}_{c u t}$ followed by a (possibly empty) block of symbols from $\mathcal{E}$. Therefore

$$
\operatorname{Card} \operatorname{Code}\left(2^{n}, \mathcal{E} \cup \mathcal{C}_{c u t}\right) \leq \sum_{m=0}^{2^{n}} \operatorname{Card} \operatorname{Code}\left(m, \mathcal{C}_{c u t}\right) \cdot \operatorname{Card} \operatorname{Code}\left(2^{n}-m, \mathcal{E}\right) .
$$

By (7) and (10),

$$
\begin{aligned}
\operatorname{Card} \operatorname{Code}\left(2^{n}, \mathcal{E} \cup \mathcal{C}_{c u t}\right) & =\left(2^{n}+1\right) \cdot O\left(2^{2 \cdot a(p) \cdot n}\right) \cdot O\left(2^{n^{2}}\right) \\
& =O\left(2^{n^{2}+(2 \cdot a(p)+1) \cdot n}\right) .
\end{aligned}
$$

Then, since $\operatorname{Span}\left(T, f, \varepsilon, 2^{n}\right) \leq \operatorname{Card} \operatorname{Code}\left(2^{n}, \mathcal{E} \cup \mathcal{C}_{\text {cut }}\right)$

$$
\begin{aligned}
\limsup _{n \rightarrow \infty} \frac{1}{n} \log \operatorname{Span}(T, f, \varepsilon, n) & \leq \limsup _{n \rightarrow \infty} \frac{1}{2^{n-1}} \log \operatorname{Span}\left(T, f, \varepsilon, 2^{n}\right) \\
& \leq \limsup _{n \rightarrow \infty} \frac{1}{2^{n-1}} \log O\left(2^{n^{2}+(2 \cdot a(p)+1) \cdot n}\right)=0 .
\end{aligned}
$$

\section{ACKNOWLEDGMENT}

The author thanks Professor Jaroslav Smítal for stimulating discussions and contributing ideas useful for this paper and Professor L'ubomír Snoha for his careful reading the paper and his helpful comments during the last stage of its preparation. 


\section{REFERENCES}

[BS] Bruckner, A. M. and Smítal, J., A characterization of $\omega$-limit sets of maps of the interval with zero topological entropy, Ergod. Th. and Dynam. Sys. 13 (1993), 7 - 19. MR 94k:26006

[FŠS] Fedorenko, V. V., Šarkovskii, A. N. and Smítal, J., Characterization of weakly chaotic maps of the interval, Proc. Amer. Math. Soc. 110 (1990), 141 - 148. MR 91a:58148

[FS] Franzová, N. and Smítal, J., Positive sequence entropy characterizes chaotic maps, Proc. Amer. Math. Soc. 112 (1991), 1083 - 1086. MR 91j:58107

[G] Goodman, T. N. T., Topological sequence entropy, Proc. London Math. Soc. 29 (1974), 331 - 350. MR 50:8482

[GH] Gottschalk, W. H. and Hedlung, G. A., Topological Dynamics, Amer. Math. Soc., 1955. MR 17:650e

[H] Harrison, J., Wandering intervals, Dynamical Systems and Turbulence (Warwick 1980), Lecture Notes in Math. 898, Springer, 1981, pp. 154 - 163. MR 83h:58059

[KS] Kolyada, S. and Snoha, L'., Topological entropy of nonautonomous dynamical systems, Random and Comp. Dynamics 4 (1996), 205 - 233. CMP 96:16

[LY] Li, T. Y. and Yorke, J. A., Period three implies chaos, Amer. Math. Monthly 82 (1975), 985 - 992. MR 52:5898

[M] Misiurewicz, M., Invariant measures for continuous transformations of $[0,1]$ with zero topological entropy, Ergodic Theory (Oberwolfach 1978), Lecture Notes in Math. 729, Springer, 1979, pp. $144-152$. MR 81a:28017

[S] Smítal, J., Chaotic functions with zero topological entropy, Trans. Amer. Math. Soc. 297 (1986), $269-281$. MR 87m:58107

Department of Mathematics, Faculty of Natural Sciences, Matej Bel University, Tajovského 40, SK-974 01 Banská Bystrica, Slovak Republic

E-mail address: hric@fpv.umb.sk 